\title{
THE DOWNSIDE AND UPSIDE BETA VALUATION IN THE VARIANCE-GAMMA MODEL
}

\author{
ROMAN V. IVANOV* \\ Laboratory of Control under Incomplete Information, V.A. Trapeznikov Institute of Control Sciences of \\ RAS, Moscow, Russian Federation \\ *Corresponding author: roivanov@yahoo.com

\begin{abstract}
The paper is aimed to assess the risks and gains of investment portfolio which relate to the impact of a particular asset. We consider the investment portfolios which consist of assets with variancegamma, gamma distributed and deterministic returns. The returns are assumed to be dependent. We derive analytical formulas for the downside and upside betas in the discussed framework. The established formulas depend on the values of a number of special mathematical functions including the values of the generalized hypergeometric ones.
\end{abstract}

\section{INTRODUCTION}

The basic monetary risk measures value at risk (see, for example, Berkowitz et al. [6], Chen and Tang [8], Ivanov [20], Stoyanov et al. [42]) and conditional value at risk (Kalinchenko et al. [22], Mafusalov and Uryasev [29], Rockafellar and Uryasev [37]) serve to assess the downward risk of the investment portfolio. But if we want to rate the influence of a specific asset on the return of the portfolio, we exploit the market beta. When we form the investment portfolio, it is necessary to estimate how the share increase or decrease for a particular asset impacts the risks and the expected profit of the portfolio. The downside beta serves to evaluate the risk size, the upside beta is used to outlay the profit.

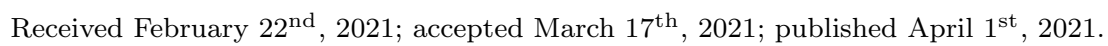

2010 Mathematics Subject Classification. 60E07, 60E08, 65C20, 91G10, 91G60, 33C20, 33C90.

Key words and phrases. downside and upside betas; variance-gamma distribution; investment portfolio; dependence; Appell function.

(C)2021 Authors retain the copyrights of their papers, and all open access articles are distributed under the terms of the Creative Commons Attribution License. 
The ideas of use the downside and upside betas go back to the paper by Roy [39] and the monograph by Markowitz [30], where it was argued that investors more care about downside losses and upside gains. In this context, Markowitz [30] suggested to use the semivariance as the basic risk measure. The semivariance beta was introduced in Hogan and Warren [19]. The advantage of downside and upside betas over the traditional ones is proposed in Ang et al. [2] and Tehir et al. [43]. The work by Estrada [13] suggests a capital asset pricing model based on the downside beta. Guy [18] presents a portfolio construction based on the assessment of the values of the upside and downside betas. Rutkowska-Ziarko and Pyke [38] introduce the downside accounting beta suggesting to use it for the measurement of the systemic risk. Altigan et al. [1] claim that the downside beta valuation is not sufficient for the asset pricing on international markets contrary to the results for the US equity market. And therefore it is required to take into account the upside one also if we want to create a general model. Advantages of downside beta-based capital asset pricing model over the traditional one are presented in Ayub et al. [4] and Post and Van Vliet [35]. In the context of the general theory, the downside beta relates to the class of loss-based risk measures which is considered in Cont et al. [9].

The variance-gamma distribution was proposed as a model for market stock returns in Madan and Seneta [28]. An utility-based option pricing theory which exploits the variance-gamma distribution was suggested in Madan and Milne [27]. The price of European call option in the variance-gamma model was derived analytically in the paper by Madan et al. [26].

There is a number of modern research papers which confirms statistically the idea of use the variancegamma distribution for the financial index modeling. Daal and Madan [12] and Finlay and Seneta [14] approve the variance-gamma model for the exchange rate simulation. Linders and Stassen [23], Moosbrucker [31] and Rathgeber et al. [36] model with the variance-gamma distribution the Dow Jones index returns. Mozumder et al. [32] consider the S\&P500 index options in the variance-gamma model. Luciano and Schoutens [25] model the S\&P500, the Nikkei225 and the Eurostoxx50 financial indexes by the variancegamma process. Luciano et al. [24] and Wallmeier and Diethelm [44] confirm the use of the variance-gamma distribution for the modeling of the US and the Swiss stock markets, respectively. Groups of various financial indices are modeled by the multivariate variance-gamma distribution in Nitithumbundit and Chan [34]. Flora and Vargiolu [15] find that the variance-gamma process is the best fit for the carbon price dynamics. Göncü et al. [16] show that the variance-gamma model fits well with the financial data of developed markets.

This paper is set to compute the downside and upside betas for the investment portfolio with the variancegamma, gamma distributed and deterministic asset returns. The gamma and deterministic returns relate to the modeling of credit risk, see Ivanov [21] and My [33]. As usually, the variance-gamma random variables are modeled as the normal mean-variance mixtures and it is supposed that the normal distributions are correlated. The paper develops the direction of research of Madan et al. [26], Ano and Ivanov [3] and 
Ivanov [20], where closed form expressions in the variance-gamma framework are derived for various targets of mathematical finance.

\section{MAin NOTATiOns}

We denote by $\gamma=\gamma(a, b)$ the gamma random variable with parameters $a, b>0$. The gamma distribution has the probability density function

$$
f(\gamma, x)=\frac{b^{a} x^{a-1} e^{-b x}}{\Gamma(a)}, \quad x>0,
$$

where $\Gamma(\chi)$ is the gamma function. It has the characteristic function

$$
\psi(\gamma, u)=\left(1-\frac{i u}{b}\right)^{-a}
$$

the mean and the variance

$$
\frac{a}{b} \quad \text { and } \quad \frac{a}{b^{2}}
$$

respectively.

By definition, the variance-gamma distribution is the mean-variance normal mixture, where the mixing density is the gamma distribution. That is, the variance-gamma random variable $H$ is defined as

$$
H=r+\theta \gamma+\sigma \sqrt{\gamma} N
$$

where $r, \theta \in \mathbb{R}, \sigma>0, N$ is the standard normally distributed random variable and the gamma random variable $\gamma$ is independent with $N$. Throughout this work, we do not assume that $\gamma$ has necessary the mean 1 , that is the identity $a=b$ is not required.

Next, we set

$$
\operatorname{sg}(\chi):= \begin{cases}1 & \text { if } \chi>0 \\ 0 & \text { if } \chi=0 \\ -1 & \text { if } \chi<0\end{cases}
$$

and use notations

$$
\mathrm{N}(\chi), \chi \in \mathbb{R}, \quad \mathrm{B}\left(\chi_{1}, \chi_{2}\right), \chi_{1}>0, \chi_{2}>0, \quad \mathrm{~K}_{\chi_{1}}\left(\chi_{2}\right), \chi_{1} \in \mathbb{R}, \chi_{2}>0
$$

for the normal distribution function, the beta function and the MacDonald function (the modified Bessel function of the second kind), respectively. The hypergeometric Gauss function is denoted as

$$
\mathrm{F}\left(\chi_{1}, \chi_{2}, \chi_{3} ; \chi_{4}\right), \chi_{1}, \chi_{2}, \chi_{3} \in \mathbb{R}, \chi_{4}<1 \text {. }
$$


Also, we discuss one of the degenerate Appell functions (or the Humbert series) which is the double sum

$$
\Phi\left(\chi_{1}, \chi_{2}, \chi_{3} ; \chi_{4}, \chi_{5}\right)=\sum_{m=0}^{\infty} \sum_{n=0}^{\infty} \frac{\left(\chi_{1}\right)_{m+n}\left(\chi_{2}\right)_{m}}{m ! n !\left(\chi_{3}\right)_{m+n}} \chi_{4}^{m} \chi_{5}^{n}
$$

with $\chi_{1}, \chi_{2}, \chi_{3}, \chi_{5} \in \mathbb{R}$ and $\left|\chi_{4}\right|<1$, where $(\chi)_{l}, l \in \mathbb{N} \cup\{0\}$, is the Pochhammer's symbol. For more information on the special mathematical functions above and relations between them, see the monographs by Bateman and Erdélyi [5] and Srivastava and Karlsson [40], the handbook by Gradshteyn and Ryzhik [17] and the papers by Chaudhry et al. [7] and Srivastava et al. [41].

\section{SETUP AND RESULTS}

Let $A_{j, t}, j=1,2, \ldots, n$, be the values of $n$ assets at time moments $t=0,1$. It is assumed that $A_{j, 0}$ are constant but $A_{j, 1}$ are random with

$$
\operatorname{Law}\left(A_{j, 1}-A_{j, 0}\right)=H_{j}
$$

where

$$
H_{j}=r_{j}+\theta_{j} \gamma_{j}+\sigma_{j} \sqrt{\gamma_{j}} N_{j}
$$

are constant, gamma or variance-gamma random variables in dependence with the values of the parameters $r_{j}, \theta_{j} \in \mathbb{R}, \sigma_{j} \geq 0$. We suggest that $\sigma_{j}>0$ for at least one $j \in\{1,2, \ldots, n\}$. It is supposed that the normal random variables $N_{j}$ and $N_{l}$ are correlated with coefficients $\rho_{j l}, j, l \leq n$. All the gamma random variables are assumed to be independent with the normal ones. We suggest that $\gamma_{j}=\kappa_{j} \gamma$, where $\gamma=\gamma(a, b)$ is gamma distributed, $\kappa_{j} \geq 0, j=1,2, \ldots, n$. Also, we set for the simplicity of formulas below that $\rho_{l m}=0$ if $\sigma_{l}=0$ or $\sigma_{m}=0$.

This model is a particular case of a more general model which is discussed in Ivanov [20]. Briefly, we assume here that the asset returns are highly dependent with each other. It agrees with the last investigations on the financial market structure, see Cont and Sirignano [10]. Together with it, the strong dependence between stocks originates to the decisions of a large investor (Cont and Wagalath [11]).

The value $I_{t}$ at time moments $t=0,1$ of the investment portfolio $x=\left(x_{1}, x_{2}, \ldots, x_{n}\right) \in \mathbb{R}^{n}$ is defined as

$$
I_{t}=\sum_{j=1}^{n} I_{j, t}
$$

where

$$
I_{j, t}=x_{j} X_{j, t}, \quad j=1,2, \ldots, n
$$


Since all $X_{j, 0}$ are constant, it is enough for the aim to evaluate the portfolio risks to discuss the random increment

$$
X=I_{1}-I_{0}=\sum_{j=1}^{n} x_{j} H_{j}
$$

of the portfolio and the random increments of the investments

$$
X_{j}=I_{j, 1}-I_{j, 0}=x_{j}\left(X_{j, 1}-X_{j, 0}\right)=x_{j} H_{j}, \quad j=1,2, \ldots, n
$$

Throughout this work we consider the downside beta $\beta^{-}$, the investment portfolio characteristics which is defined as

$$
\beta^{-}=\frac{\mathrm{E}\left[\left(X_{j}-\mathrm{E} X_{j}\right)(X-\mathrm{E} X) I_{\{X \leq u\}}\right]}{\mathrm{E}\left[(X-\mathrm{E} X)^{2} I_{\{X \leq u\}}\right]}, \quad u \in \mathbb{R} .
$$

Taking into account the value of $\beta^{-}$, one could analyze is it expedient to put the size $x_{j}$ into the asset $j$ or not. For example, if $\beta^{-} \gg 0$, it means that the investment $x_{j} X_{j, 0}$ accelerates the downside risk of the portfolio substantially. Together with the downside beta, we discuss the upside beta

$$
\beta^{+}=\frac{\mathrm{E}\left[\left(X_{j}-\mathrm{E} X_{j}\right)(X-\mathrm{E} X) I_{\{X \geq u\}}\right]}{\mathrm{E}\left[(X-\mathrm{E} X)^{2} I_{\{X \geq u\}}\right]}, \quad u \in \mathbb{R} .
$$

The upside beta shows the impact of the investment $x_{j}$ on the potential earnings of the investment portfolio.

To introduce the results, now we suggest some auxiliary abbreviations. Let

$$
\begin{array}{cc}
h_{j}=x_{j}\left(r_{j}+\frac{\theta_{j} a_{j}}{b_{j}}\right), \quad h=\sum_{l=1}^{n} h_{l}, \quad \widehat{s}=\sum_{l=1}^{n} x_{l} r_{l} \quad \widehat{u}=u-\widehat{s}, \\
s_{1}=\sum_{l=1}^{n} x_{l} \theta_{l} \kappa_{l}, \quad s_{2}=\sum_{l=1}^{n} \rho_{j l} x_{l} \sigma_{l} \sqrt{\kappa_{l}}, \quad s_{3}=\sqrt{\sum_{m, l=1}^{n} \rho_{m l} x_{m} \sigma_{m} x_{l} \sigma_{l} \sqrt{\kappa_{m} \kappa_{l}}}, \\
s=\frac{\widehat{u} \sqrt{s_{1}^{2}+2 b s_{3}^{2}}}{s_{3}\left|s_{3}\right|}, & q=-\frac{\operatorname{sg}\left(s_{3}\right) s_{1}}{\sqrt{s_{1}^{2}+2 b s_{3}^{2}}} .
\end{array}
$$

Next, we set

$$
Y_{l}=x_{l} \sigma_{l} \sqrt{\gamma_{l}} N_{l}, \quad x_{l}, \sigma_{l} \neq 0
$$

The lemma below computes the value of the expectation

$$
f_{1}=\mathrm{E}\left(Y_{j} \sum_{l=1}^{n} Y_{l} I_{\left\{\sum_{l=1}^{n} Y_{l} \leq u-\sum_{l=1}^{n} x_{l}\left(r_{l}+\theta_{l} \gamma_{l}\right)\right\}}\right) .
$$

Lemma 3.1. If $x_{j}, \sigma_{j} \neq 0$, the expectation

$$
f_{1}=\frac{x_{j} \sigma_{j} s_{2} b^{a} \sqrt{\kappa_{j}}}{s_{3} \Gamma(a)}\left(s_{3} \Lambda(\widehat{u})-\frac{\exp \left(\frac{\widehat{u} s_{1}}{s_{3}^{2}}\right)}{\sqrt{2 \pi}}\left(\widehat{u} \Theta(\widehat{u}, 0)-s_{1} \Theta(\widehat{u}, 1)\right)\right),
$$


where

$$
\begin{aligned}
\Lambda(\widehat{u}) & =\frac{\Gamma\left(a+\frac{3}{2}\right)}{b^{a+1} \sqrt{2 \pi}}\left(\frac{\mathrm{B}\left(\frac{1}{2}, a+1\right)}{\sqrt{2}}-\frac{s_{1}}{s_{3} \sqrt{b}} \mathrm{~F}\left(a+\frac{3}{2}, \frac{1}{2}, \frac{3}{2} ;-\frac{s_{1}^{2}}{2 b s_{3}^{2}}\right)\right) I_{\{\widehat{u}=0\}}+ \\
& +\frac{|s|^{a+\frac{1}{2}} e^{s}(1+q)^{a+1}}{b^{a+1} \sqrt{2 \pi}}\left(\left(|s| \mathrm{K}_{a+\frac{3}{2}}(|s|)+s \mathrm{~K}_{a+\frac{1}{2}}(|s|)\right) \widehat{\Phi}(0)-\right. \\
& \left.-(1+q) s \mathrm{~K}_{a+\frac{1}{2}}(|s|) \widehat{\Phi}(1)\right) I_{\{\widehat{u} \neq 0\}}
\end{aligned}
$$

and

$$
\begin{aligned}
& \Theta(\widehat{u}, j)=\Gamma\left(a+\frac{1}{2}+j\right)\left(\frac{2 s_{3}^{2}}{s_{1}^{2}+2 b s_{3}^{2}}\right)^{a+\frac{1}{2}+j} I_{\{\widehat{u}=0\}}+ \\
& +2\left(\frac{\widehat{u}^{2}}{s_{1}^{2}+2 b s_{3}^{2}}\right)^{\frac{a+j}{2}+\frac{1}{4}} \mathrm{~K}_{a+j+\frac{1}{2}}\left(\frac{|\widehat{u}| \sqrt{s_{1}^{2}+2 b s_{3}^{2}}}{s_{3}^{2}}\right) I_{\{\widehat{u} \neq 0\}}
\end{aligned}
$$

with

$$
\widehat{\Phi}(j)=\mathrm{B}(a+1+j, 1) \Phi\left(a+1+j,-a, a+2+j ; \frac{1+q}{2},-s(1+q)\right) .
$$

Next, we discuss the expectation

$$
f_{2}(\zeta, \alpha)=\mathrm{E}\left(\gamma_{j}^{\zeta}\left(\sum_{l=1}^{n} x_{l} \theta_{l} \gamma_{l}\right)^{\alpha} Y_{j} I_{\left\{\sum_{l=1}^{n} Y_{l} \leq u-\sum_{l=1}^{n} x_{l}\left(r_{l}+\theta_{l} \gamma_{l}\right)\right\}}\right) .
$$

for $\zeta, \alpha \in \mathbb{N} \cup\{0\}$.

Lemma 3.2. When $x_{j}, \sigma_{j} \neq 0$, we have that

$$
\begin{aligned}
& f_{2}(\zeta, \alpha)=-\frac{s_{2} x_{j} \sigma_{j} s_{1}^{\alpha} b^{a} \kappa_{j}^{\zeta+\frac{1}{2}}}{\Gamma(a) \sqrt{\pi}}\left(\frac{2^{\zeta+\alpha+a} s_{3}^{2(\zeta+\alpha+a)} \Gamma\left(\alpha+a+\frac{1}{2}\right)}{\left(s_{1}^{2}+2 b s_{3}^{2}\right)^{\zeta+\alpha+a+\frac{1}{2}}} I_{\{\widehat{u}=0\}}+\right. \\
& \left.+\frac{\exp \left(\frac{\widehat{u} s_{1}}{s_{3}^{2}}\right) \sqrt{2}}{s_{3}}\left(\frac{\widehat{u}^{2}}{s_{1}^{2}+2 b s_{3}^{2}}\right)^{\frac{\zeta+\alpha+a}{2}+\frac{1}{4}} \mathrm{~K}_{\zeta+\alpha+a+\frac{1}{2}}\left(\frac{|\widehat{u}| \sqrt{s_{1}^{2}+2 b s_{3}^{2}}}{s_{3}^{2}}\right) I_{\{\widehat{u} \neq 0\}}\right) .
\end{aligned}
$$

Also, we calculate the function

$$
f_{3}(\zeta, \alpha)=\mathrm{E}\left(\gamma_{j}^{\zeta}\left(\sum_{l=1}^{n} x_{l} \theta_{l} \gamma_{l}\right)^{\alpha} I_{\left\{\sum_{l=1}^{n} Y_{l} \leq u-\sum_{l=1}^{n} x_{l}\left(r_{l}+\theta_{l} \gamma_{l}\right)\right\}}\right) .
$$


Lemma 3.3. Let $\zeta, \alpha \in \mathbb{N} \cup\{0\}$. Then

$$
\begin{aligned}
& f_{3}(\zeta, \alpha)=\frac{\kappa_{j}^{\zeta} s_{1}^{\alpha} \Gamma\left(\zeta+\alpha+a+\frac{1}{2}\right)}{\Gamma(a) b^{\zeta+\alpha} \sqrt{2 \pi}} I_{\{\widehat{u}=0\}}\left(\frac{\mathrm{B}\left(\frac{1}{2}, \zeta+\alpha+a\right)}{\sqrt{2}}-\frac{s_{1}}{s_{3} \sqrt{b}} \times\right. \\
& \left.\times \mathrm{F}\left(\zeta+\alpha+a+\frac{1}{2}, \frac{1}{2}, \frac{3}{2} ;-\frac{s_{1}^{2}}{2 b s_{3}^{2}}\right)\right)+\frac{\kappa_{j}^{\zeta}|s|^{\zeta+\alpha+a-\frac{1}{2}}(1+q)^{\zeta+\alpha+a} I_{\{\widehat{u} \neq 0\}}}{\Gamma(a) b^{\zeta+\alpha} e^{-s} s_{1}^{-\alpha} \sqrt{2 \pi}} \times \\
& \times\left(\mathrm{B}(\zeta+\alpha+a, 1)\left(|s| \mathrm{K}_{\zeta+\alpha+a+\frac{1}{2}}(|s|)+s \mathrm{~K}_{\zeta+\alpha+a-\frac{1}{2}}(|s|)\right) \tilde{\Phi}(0)-\right. \\
& \left.-(1+q) s \mathrm{~B}(\zeta+\alpha+a+1,1) \mathrm{K}_{\zeta+\alpha+a-\frac{1}{2}}(|s|) \tilde{\Phi}(1)\right)
\end{aligned}
$$

where

$$
\begin{aligned}
& \tilde{\Phi}(j)= \\
= & \Phi\left(\zeta+\alpha+a+j, 1-\zeta-\alpha-a, \zeta+\alpha+a+1+j ; \frac{1+q}{2},-s(1+q)\right) .
\end{aligned}
$$

Set

$$
f_{4}(\zeta, \alpha)=\mathrm{E}\left(\gamma_{j}^{\zeta}\left(\sum_{l=1}^{n} x_{l} \theta_{l} \gamma_{l}\right)^{\alpha} \sum_{l=1}^{n} Y_{l} I_{\left\{\sum_{l=1}^{n} Y_{l} \leq u-\sum_{l=1}^{n} x_{l}\left(r_{l}+\theta_{l} \gamma_{l}\right)\right\}}\right)
$$

for $\zeta, \alpha \in \mathbb{N} \cup\{0\}$.

Lemma 3.4. The expectation

$$
\begin{aligned}
& f_{4}(\zeta, \alpha)=-\frac{s_{3} b^{a} s_{1}^{\alpha} \kappa_{j}^{\zeta}}{\Gamma(a) \sqrt{2 \pi}}\left(2 e^{\frac{\widehat{u} s_{1}}{s_{3}^{2}}} \mathrm{~K}_{\zeta+\alpha+a+\frac{1}{2}}\left(\frac{|\widehat{u}| \sqrt{s_{1}^{2}+2 b s_{3}^{2}}}{s_{3}^{2}}\right) I_{\{\widehat{u}=0\}} \times\right. \\
& \left.\left(\frac{\widehat{u}^{2}}{s_{1}^{2}+2 b s_{3}^{2}}\right)^{\frac{\zeta+\alpha+a}{2}+\frac{1}{4}}+\Gamma\left(\zeta+\alpha+a+\frac{1}{2}\right)\left(\frac{2 s_{3}^{2}}{s_{1}^{2}+2 b s_{3}^{2}}\right)^{\zeta+\alpha+a+\frac{1}{2}} I_{\{\widehat{u} \neq 0\}}\right) .
\end{aligned}
$$

Finally, set

$$
f_{5}=\mathrm{E}\left(\left(\sum_{l=1}^{n} Y_{l}\right)^{2} I_{\left\{\sum_{l=1}^{n} Y_{l} \leq u-\sum_{l=1}^{n} x_{l}\left(r_{l}+\theta_{l} \gamma_{l}\right)\right\}}\right) .
$$

Lemma 3.5. Let the functions $\Theta(\widehat{u}, j)$ and $\Lambda(\widehat{u})$ be defined in Lemma 3.1. Then

$$
\begin{gathered}
f_{5}=\frac{s_{3} b^{a}}{\Gamma(a)}\left(\frac{s_{1}}{\sqrt{2 \pi}} \Theta(0,1)+s_{3} \Lambda(0)\right) I_{\{\widehat{u}=0\}}+ \\
+\frac{s_{3} b^{a}}{\Gamma(a)}\left(s_{3} \Lambda(\widehat{u})+\frac{\exp \left(\frac{\widehat{u} s_{1}}{s_{3}^{2}}\right)}{\sqrt{2 \pi}}\left(s_{1} \Theta(\widehat{u}, 1)-\widehat{u} \Theta(\widehat{u}, 0)\right)\right) I_{\{\widehat{u} \neq 0\}} .
\end{gathered}
$$

The proofs of Lemmas 3.1-3.5 are placed in Section 4.

Now can introduce the main results of the paper. The theorem below gives us an analytical expression for the value of the downside beta. 
Theorem 3.1. The downside beta

$$
\beta^{-}=\frac{\beta_{n}^{-}}{\beta_{d}^{-}}
$$

with

$$
\begin{aligned}
& \beta_{n}^{-}=x_{j}\left[r_{j}\left(\widehat{s} f_{3}(0,0)+f_{3}(0,1)+f_{4}(0,0)\right)+\theta_{j}\left(\widehat{s} f_{3}(1,0)+\right.\right. \\
& \left.\left.+f_{3}(1,1)+f_{4}(1,0)\right)\right]+\widehat{s} f_{2}(0,0)+f_{2}(0,1)+f_{1}-h_{j}\left(\widehat{s} f_{3}(0,0)+\right. \\
& \left.+f_{3}(0,1)+f_{4}(0,0)-h f_{3}(0,0)\right)-h\left[x_{j}\left(r_{j} f_{3}(0,0)+\theta_{j} f_{3}(1,0)\right)+f_{2}(0,0)\right]
\end{aligned}
$$

and

$$
\begin{aligned}
\beta_{d}^{-} & =\widehat{s}^{2} f_{3}(0,0)+2 \widehat{s} f_{3}(0,1)+f_{3}(0,2)+2 \widehat{s} f_{4}(0,0)+2 f_{4}(0,1)+ \\
& +f_{5}-2 h\left(\widehat{s} f_{3}(0,0)+f_{3}(0,1)+f_{4}(0,0)\right)+h^{2} f_{3}(0,0),
\end{aligned}
$$

where the expectations $f_{1}, f_{2}, f_{3}, f_{4}, f_{5}$ are computed in Lemmas 3.1-3.5, respectively.

Let

$$
\begin{gathered}
\widehat{h}_{j}=x_{j} \sum_{l=1}^{n} x_{l}\left(r_{j}\left(r_{l}+\frac{\theta_{l} a_{l}}{b_{l}}\right)+\theta_{j}\left(\frac{r_{l} a_{j}}{b_{j}}+\right.\right. \\
\left.\left.+\frac{\theta_{l} \kappa_{l} \kappa_{j}(a+1) a}{b^{2}}\right)+\sigma_{j}\left(r_{l}+\frac{\sigma_{l} \rho_{l j} a \sqrt{\kappa_{l} \kappa_{j}}}{b}\right)\right)
\end{gathered}
$$

and

$$
\begin{aligned}
\widehat{h} & =\sum_{l, m=1}^{n} x_{l} x_{m}\left(r_{l}\left(r_{m}+\frac{\theta_{m} a_{m}}{b_{m}}\right)+\theta_{l}\left(\frac{r_{m} a_{l}}{b_{l}}+\right.\right. \\
& \left.\left.+\frac{\theta_{m} \kappa_{l} \kappa_{m} a(a+1)}{b^{2}}\right)+\frac{\sigma_{l} \sigma_{m} \rho_{l m} a \sqrt{\kappa_{l} \kappa_{m}}}{b}\right) .
\end{aligned}
$$

The next theorem derives the size of the upside beta.

Theorem 3.2. The upside beta

$$
\beta^{+}=\frac{\widehat{h}_{j}-h_{j} h-\beta_{n}^{-}}{\widehat{h}-h^{2}-\beta_{d}^{-}},
$$

where $\beta_{n}^{-}$and $\beta_{d}^{-}$are defined in (3.9) and (3.10).

The proofs of Theorems 3.1 and 3.2 are given in Section 4 .

The following example considers the case of the investment portfolio which consists of three assets and two of them are risk-free and low risk ones. 
Example 3.1. Assume that $n=3, H_{1}=r_{1}, H_{2}=r_{2}+\theta_{2} \gamma, H_{3}=r_{3}+\theta_{3} \gamma+\sigma_{3} \sqrt{\gamma} N_{3}$ and $j=2$. Then $h_{2}=x_{2}\left(r_{2}+\frac{\theta_{2} a_{2}}{b_{2}}\right), h=r_{1}+\sum_{l=2}^{3} x_{l}\left(r_{l}+\frac{\theta_{l} a_{l}}{b_{l}}\right), \widehat{s}=\sum_{l=1}^{3} x_{l} r_{l}, \widehat{u}=u-\widehat{s}, s_{1}=\sum_{l=2}^{3} x_{l} \theta_{l}, s_{2}=0$, $s_{3}=\sigma_{3}\left|x_{3}\right|$,

$$
\begin{gathered}
\widehat{h}_{2}= \\
=x_{2}\left(x_{1}\left(r_{2} r_{1}+\frac{\theta_{2} r_{1} a}{b}\right)+\sum_{l=2}^{3} x_{l}\left(r_{2}\left(r_{l}+\frac{\theta_{l} a}{b}\right)+\theta_{2}\left(\frac{r_{l} a}{b}+\frac{\theta_{l}(a+1) a}{b^{2}}\right)\right)\right), \\
\widehat{h}=\sum_{l, m=1}^{3} x_{l} x_{m}\left(r_{l}\left(r_{m}+\frac{\theta_{m} I_{\{m \neq 1\}} a}{b}\right)+\right. \\
\left.+\theta_{l} I_{\{l \neq 1\}}\left(\frac{r_{m} a}{b}+\frac{\theta_{m} I_{\{m \neq 1\}} a(a+1)}{b^{2}}\right)\right)+\frac{\sigma_{3}^{2} a}{b}
\end{gathered}
$$

$f_{1} \equiv 0, f_{2}(\zeta, \alpha) \equiv 0$

$$
\begin{aligned}
& \beta_{n}^{-}=x_{j}\left(r_{j}\left(\widehat{s} f_{3}(0,0)+f_{3}(0,1)+f_{4}(0,0)\right)+\right. \\
+ & \left.\theta_{j}\left(\widehat{s} f_{3}(1,0)+f_{3}(1,1)+f_{4}(1,0)\right)\right)-h_{j}\left(\widehat{s} f_{3}(0,0)+f_{3}(0,1)+\right. \\
+ & \left.f_{4}(0,0)-h f_{3}(0,0)\right)-h\left(x_{j}\left(r_{j} f_{3}(0,0)+\theta_{j} f_{3}(1,0)\right)\right),
\end{aligned}
$$

where $f_{3}, f_{4}$ are determined by Lemma 3.3, Lemma 3.4 and $\beta_{d}^{-}$is calculated in (3.10).

Example 3.2 discusses the case when there are $n$ assets in the portfolio and they are the medium dependent between each other.

Example 3.2. Let $\gamma_{1} \equiv \gamma_{2} \equiv \ldots \equiv \gamma_{n} \equiv \gamma$ and $\rho_{l m}=0, l \neq m$. We have that $h_{j}=x_{j}\left(r_{j}+\frac{\theta_{j} a}{b}\right)$, $h=\sum_{l=1}^{n} h_{l}, \widehat{s}=\sum_{l=1}^{n} x_{l} r_{l}, \widehat{u}=u-\widehat{s}, s_{1}=\sum_{l=1}^{n} x_{l} \theta_{l}, s_{2}=x_{j} \sigma_{j}, s_{3}=\sqrt{\sum_{l=1}^{n} x_{l}^{2} \sigma_{l}^{2}}$,

$$
\begin{aligned}
& \widehat{h}_{j}=x_{j}\left(\frac{x_{j} \sigma_{j}^{2} a}{b}+\sum_{l=1}^{n} x_{l}\left(r_{j}\left(r_{l}+\frac{\theta_{l} a}{b}\right)+\theta_{j}\left(\frac{r_{l} a}{b}+\frac{\theta_{l}(a+1) a}{b^{2}}\right)+\sigma_{j} r_{l}\right)\right), \\
& \widehat{h}=\sum_{l, m=1}^{n} x_{l} x_{m}\left(r_{l}\left(r_{m}+\frac{\theta_{m} a}{b}\right)+\theta_{l}\left(\frac{r_{m} a}{b}+\frac{\theta_{m} a(a+1)}{b^{2}}\right)\right)+\frac{a}{b} \sum_{l=1}^{n} x_{l}^{2} \sigma_{l}^{2}
\end{aligned}
$$

and $\beta_{n}^{-}, \beta_{d}^{-}$are computed with respect to (3.9), (3.10).

\section{Proofs}

Proof of Lemma 3.1. It is easy to notice that $\left(Y_{j}, \sum_{l=1}^{n} Y_{l} \mid \gamma_{1}, \gamma_{2}, \ldots, \gamma_{n}\right)$ is a Gaussian vector with the covariance matrix

$$
\left(\begin{array}{cc}
\left(x_{j} \sigma_{j}\right)^{2} \gamma_{j} & \sum_{l=1}^{n} \rho_{j l} x_{j} \sigma_{j} x_{l} \sigma_{l} \sqrt{\gamma_{j} \gamma_{l}} \\
\sum_{l=1}^{n} \rho_{j l} x_{j} \sigma_{j} x_{l} \sigma_{l} \sqrt{\gamma_{j} \gamma_{l}} & \sum_{m, l=1}^{n} \rho_{m l} x_{m} \sigma_{m} x_{l} \sigma_{l} \sqrt{\gamma_{m} \gamma_{l}}
\end{array}\right) .
$$




\section{Hence}

$$
\operatorname{Law}\left(Y_{j}, \sum_{l=1}^{n} Y_{l} \mid \gamma_{1}, \gamma_{2}, \ldots, \gamma_{n}\right)=\operatorname{Law}\left(Y_{j}, \tilde{Y}\right)
$$

where $\tilde{Y}=\sigma_{\tilde{Y}} \tilde{N}$ with

$$
\sigma_{\tilde{Y}}=\sqrt{\sum_{m, l=1}^{n} \rho_{m l} x_{m} \sigma_{m} x_{l} \sigma_{l} \sqrt{\gamma_{m} \gamma_{l}}}
$$

and the standard normal random variables $N_{j}$ and $\tilde{N}$ are correlated with the coefficient

$$
\rho_{j \tilde{Y}}=\frac{\sum_{l=1}^{n} \rho_{j l} x_{j} \sigma_{j} x_{l} \sigma_{l} \sqrt{\gamma_{j} \gamma_{l}}}{x_{j} \sigma_{j} \sqrt{\gamma_{j}} \sqrt{\sum_{m, l=1}^{n} \rho_{m l} x_{m} \sigma_{m} x_{l} \sigma_{l} \sqrt{\gamma_{m} \gamma_{l}}}} .
$$

Set

$$
\widehat{\sigma}_{j}=x_{j} \sigma_{j} \sqrt{\gamma_{j}}
$$

and

$$
\tilde{u}=u-\sum_{l=1}^{n} x_{l}\left(r_{l}+\theta_{l} \gamma_{l}\right) .
$$

Then

$$
\begin{aligned}
& \mathrm{E}\left(Y_{j} \sum_{l=1}^{n} Y_{l} I_{\left\{\sum_{l=1}^{n} Y_{l} \leq u-\sum_{l=1}^{n} x_{l}\left(r_{l}+\theta_{l} \gamma_{l}\right)\right\}} \mid \gamma_{1}, \gamma_{2}, \ldots, \gamma_{n}\right)= \\
= & \mathrm{E}\left(Y_{j} \tilde{Y} I_{\{\tilde{Y} \leq \tilde{u}\}} \mid \gamma_{1}, \gamma_{2}, \ldots, \gamma_{n}\right)=\int_{-\infty}^{\tilde{u}} \int_{-\infty}^{\infty} \frac{x y}{2 \pi \sigma_{\tilde{Y}} \widehat{\sigma}_{j} \sqrt{1-\rho_{j \tilde{Y}}^{2}}} \times \\
\times & \exp \left(-\frac{1}{2\left(1-\rho_{j \tilde{Y}}^{2}\right)}\left[\frac{x^{2}}{\sigma_{\tilde{Y}}^{2}}-2 \rho_{j \tilde{Y}} \frac{x y}{\sigma_{\tilde{Y}} \widehat{\sigma}_{j}}+\frac{y^{2}}{\widehat{\sigma}_{j}^{2}}\right]\right) d y d x
\end{aligned}
$$


and since

$$
\begin{aligned}
& \int_{-\infty}^{\infty} y \exp \left(-\frac{1}{2\left(1-\rho_{j \tilde{Y}}^{2}\right)}\left[\frac{y^{2}}{\widehat{\sigma}_{j}^{2}}-2 \rho_{j \tilde{Y}} \frac{x y}{\sigma_{\tilde{Y}} \widehat{\sigma}_{j}}\right]\right) d y= \\
& =\exp \left(\frac{\left(x \rho_{j \tilde{Y}}\right)^{2}}{2 \sigma_{\tilde{Y}}^{2}\left(1-\rho_{j \tilde{Y}}^{2}\right)}\right) \int_{-\infty}^{\infty} y \exp \left(-\frac{1}{2\left(1-\rho_{j \tilde{Y}}^{2}\right)}\left[\frac{y}{\widehat{\sigma}_{j}}-\frac{x \rho_{j \tilde{Y}}}{\sigma_{\tilde{Y}}}\right]^{2}\right) d y= \\
& =\widehat{\sigma}_{j}^{2} \exp \left(\frac{\left(x \rho_{j \tilde{Y}}\right)^{2}}{2 \sigma_{\tilde{Y}}^{2}\left(1-\rho_{j \tilde{Y}}^{2}\right)}\right)\left(\int_{-\infty}^{\infty}\left(y-\frac{x \rho_{j \tilde{Y}}}{\sigma_{\tilde{Y}}}\right) \times\right. \\
& \times \exp \left(-\frac{1}{2\left(1-\rho_{j \tilde{Y}}^{2}\right)}\left[y-\frac{x \rho_{j \tilde{Y}}}{\sigma_{\tilde{Y}}^{2}}\right]^{2} d y+\frac{x \rho_{j \tilde{Y}}}{\sigma_{\tilde{Y}}} \times\right. \\
& \times \int_{-\infty}^{\infty} \exp \left(-\frac{1}{2\left(1-\rho_{j \tilde{Y}}^{2}\right)}\left[y-\frac{x \rho_{j \tilde{Y}}}{\sigma_{\tilde{Y}}}\right]^{2} d y\right)= \\
& =\exp \left(\frac{\left(x \rho_{j \tilde{Y}}\right)^{2}}{2 \sigma_{\tilde{Y}}^{2}\left(1-\rho_{j \tilde{Y}}^{2}\right)}\right) \frac{x \rho_{j \tilde{Y}} \widehat{\sigma}_{j}^{2}}{\sigma_{\tilde{Y}}} \sqrt{2 \pi\left(1-\rho_{j \tilde{Y}}^{2}\right)}
\end{aligned}
$$

we have that

$$
\begin{aligned}
& \mathrm{E}\left(Y_{j} \sum_{l=1}^{n} Y_{l} I_{\left\{\sum_{l=1}^{n} Y_{l} \leq u-\sum_{l=1}^{n} x_{l}\left(r_{l}+\theta_{l} \gamma_{l}\right)\right\}} \mid \gamma_{1}, \gamma_{2}, \ldots, \gamma_{n}\right)=\int_{-\infty}^{\tilde{u}} \frac{x^{2} \rho_{j \tilde{Y}} \widehat{\sigma}_{j}}{\sigma_{\tilde{Y}}^{2} \sqrt{2 \pi}} \times \\
& \times \exp \left(-\frac{x^{2}}{2 \sigma_{\tilde{Y}}^{2}}\right) d x=-\frac{\rho_{j \tilde{Y}} \widehat{\sigma}_{j}}{\sqrt{2 \pi}} \int_{-\infty}^{\tilde{u}} x d \exp \left(-\frac{x^{2}}{2 \sigma_{\tilde{Y}}^{2}}\right)= \\
& =-\frac{\rho_{j \tilde{Y}} \widehat{\sigma}_{j}}{\sqrt{2 \pi}} \tilde{u} \exp \left(-\frac{\tilde{u}^{2}}{2 \sigma_{\tilde{Y}}^{2}}\right)+\frac{\rho_{j \tilde{Y}} \widehat{\sigma}_{j}}{\sqrt{2 \pi}} \int_{-\infty}^{\tilde{u}} \exp \left(-\frac{x^{2}}{2 \sigma_{\tilde{Y}}^{2}}\right) d x= \\
& =-\frac{\rho_{j \tilde{Y}} \widehat{\sigma}_{j}}{\sqrt{2 \pi}} \tilde{u} \exp \left(-\frac{\tilde{u}^{2}}{2 \sigma_{\tilde{Y}}^{2}}\right)+\rho_{j \tilde{Y}} \sigma_{\tilde{Y}} \widehat{\sigma}_{j} \mathrm{~N}\left(\frac{\tilde{u}}{\sigma_{\tilde{Y}}}\right) \text {. }
\end{aligned}
$$

Next, one can observe that

$$
\sigma_{\tilde{Y}}=\sqrt{\gamma \sum_{m, l=1}^{n} \rho_{m l} x_{m} \sigma_{m} x_{l} \sigma_{l} \sqrt{\kappa_{m} \kappa_{l}}}
$$

and

$$
\tilde{u}=u-\sum_{l=1}^{n} x_{l} r_{l}-\gamma \sum_{l=1}^{n} x_{l} \theta_{l} \kappa_{l}
$$

Moreover, we have that

$$
\rho_{j \tilde{Y}}=\frac{\gamma \sum_{l=1}^{n} \rho_{j l} x_{j} \sigma_{j} x_{l} \sigma_{l} \sqrt{\kappa_{j} \kappa_{l}}}{x_{j} \sigma_{j} \sqrt{\kappa_{j} \gamma} \sqrt{\gamma \sum_{m, l=1}^{n} \rho_{m l} x_{m} \sigma_{m} x_{l} \sigma_{l} \sqrt{\kappa_{m} \kappa_{l}}}}
$$


and

$$
\widehat{\sigma}_{j}=x_{j} \sigma_{j} \sqrt{\kappa_{j} \gamma}
$$

Set

$$
\begin{aligned}
& \widehat{u}=u-\sum_{l=1}^{n} x_{l} r_{l}, s_{1}=\sum_{l=1}^{n} x_{l} \theta_{l} \kappa_{l}, s_{2}=\sum_{l=1}^{n} \rho_{j l} x_{l} \sigma_{l} \sqrt{\kappa_{l}}, \\
& s_{3}=\sqrt{\sum_{m, l=1}^{n} \rho_{m l} x_{m} \sigma_{m} x_{l} \sigma_{l} \sqrt{\kappa_{m} \kappa_{l}}} .
\end{aligned}
$$

Then

$$
\sigma_{\tilde{Y}}=s_{3} \sqrt{\gamma}, \quad \tilde{u}=\widehat{u}-s_{1} \gamma, \quad \rho_{j \tilde{Y}}=\frac{s_{2}}{s_{3}}
$$

and we get from (4.6) that

$$
\begin{aligned}
& \mathrm{E}\left(Y_{j} \sum_{l=1}^{n} Y_{l} I_{\left\{\sum_{l=1}^{n} Y_{l} \leq u-\sum_{l=1}^{n} x_{l}\left(r_{l}+\theta_{l} \gamma_{l}\right)\right\}}\right)= \\
= & x_{j} \sigma_{j} \frac{s_{2}}{s_{3}} \sqrt{\kappa_{j}}\left(s_{3} \int_{0}^{\infty} g \mathrm{~N}\left(\frac{\widehat{u}-s_{1} g}{s_{3} \sqrt{g}}\right) f(\gamma, g) d g-\right. \\
- & \left.\frac{1}{\sqrt{2 \pi}} \int_{0}^{\infty}\left(\widehat{u}-s_{1} g\right) \sqrt{g} \exp \left(-\frac{\left(\widehat{u}-s_{1} g\right)^{2}}{2 s_{3}^{2} g}\right) f(\gamma, g) d g\right)= \\
= & \frac{x_{j} \sigma_{j} s_{2} b^{a} \sqrt{\kappa_{j}}}{s_{3} \Gamma(a)}\left(s_{3} \int_{0}^{\infty} g^{a} \mathrm{~N}\left(\frac{\widehat{u}-s_{1} g}{s_{3} \sqrt{g}}\right) \exp (-b g) d g-\right. \\
& \left.-\frac{\exp \left(\frac{\widehat{u} s_{1}}{s_{3}^{2}}\right)}{\sqrt{2 \pi}} \int_{0}^{\infty}\left(\widehat{u}-s_{1} g\right) g^{a-\frac{1}{2}} \exp \left(-\frac{\widehat{u}^{2}+\left(s_{1} g\right)^{2}}{2 s_{3}^{2} g}-b g\right) d g\right) .
\end{aligned}
$$

First,

$$
\begin{aligned}
& \int_{0}^{\infty} g^{a \pm \frac{1}{2}} \exp \left(-\frac{\widehat{u}^{2}}{2 s_{3}^{2} g}-\frac{s_{1}^{2}+2 b s_{3}^{2}}{2 s_{3}^{2}} g\right) d g= \\
= & 2\left(\frac{\widehat{u}^{2}}{s_{1}^{2}+2 b s_{3}^{2}}\right)^{\frac{a+1}{2} \pm \frac{1}{4}} \mathrm{~K}_{a+1 \pm \frac{1}{2}}\left(\frac{|\widehat{u}| \sqrt{s_{1}^{2}+2 b s_{3}^{2}}}{s_{3}^{2}}\right)
\end{aligned}
$$

with respect to the formula 3.471.9 from Gradshteyn and Ryzhik [17] if $\widehat{u} \neq 0$. When $\widehat{u}=0$,

$$
\begin{aligned}
& \int_{0}^{\infty} g^{a \pm \frac{1}{2}} \exp \left(-\frac{s_{1}^{2}+2 b s_{3}^{2}}{2 s_{3}^{2}} g\right) d g= \\
& =\Gamma\left(a+1 \pm \frac{1}{2}\right)\left(\frac{2 s_{3}^{2}}{s_{1}^{2}+2 b s_{3}^{2}}\right)^{a+1 \pm \frac{1}{2}} .
\end{aligned}
$$

Next, the integral

$$
I=\int_{0}^{\infty} g^{a} \mathrm{~N}\left(\frac{\widehat{u}-s_{1} g}{s_{3} \sqrt{g}}\right) \exp (-b g) d g
$$


is quite similar to the one at the bottom of p.207 of Ivanov and Ano [3]. If $\widehat{u}=0$,

$$
I=\frac{\Gamma\left(a+\frac{3}{2}\right)}{b^{a+1} \sqrt{2 \pi}}\left(\frac{\mathrm{B}\left(\frac{1}{2}, a+1\right)}{\sqrt{2}}-\frac{s_{1}}{s_{3} \sqrt{b}} \mathrm{~F}\left(a+\frac{3}{2}, \frac{1}{2}, \frac{3}{2} ;-\frac{s_{1}^{2}}{2 b s_{3}^{2}}\right)\right)
$$

due to Case 2.2, p.208 of Ivanov and Ano [3]. When $\widehat{u} \neq 0$,

$$
\begin{aligned}
& I=\frac{|s|^{a+\frac{1}{2}} e^{s}(1+q)^{a+1}}{b^{a+1} \sqrt{2 \pi}}\left(\mathrm { B } ( a + 1 , 1 ) \left(|s| \mathrm{K}_{a+\frac{3}{2}}(|s|)+\right.\right. \\
& \left.s \mathrm{~K}_{a+\frac{1}{2}}(|s|)\right) \Phi\left(a+1,-a, a+2 ; \frac{1+q}{2},-s(1+q)\right)- \\
& \left.(1+q) s \mathrm{~B}(a+2,1) \mathrm{K}_{a+\frac{1}{2}}(|s|) \Phi\left(a+2,-a, a+3 ; \frac{1+q}{2},-s(1+q)\right)\right),
\end{aligned}
$$

where $s=\frac{\widehat{u} \sqrt{s_{1}^{2}+2 b s_{3}^{2}}}{s_{3}\left|s_{3}\right|}$ and $q=-\frac{\operatorname{sg}\left(s_{3}\right) s_{1}}{\sqrt{s_{1}^{2}+2 b s_{3}^{2}}}$, with respect to Case 3.2, p.210 of Ivanov and Ano [3]. Hence we get (3.4) from (4.7)-(4.11).

Proof of Lemma 3.2. Keeping in mind (4.1), we get that

$$
\begin{aligned}
& \mathrm{E}\left(Y_{j} I_{\left\{\sum_{l=1}^{n} Y_{l} \leq u-\sum_{l=1}^{n} x_{l}\left(r_{l}+\theta_{l} \gamma_{l}\right)\right\}} \mid \gamma_{1}, \gamma_{2}, \ldots, \gamma_{n}\right)= \\
& =\mathrm{E}\left(Y_{j} I_{\{\tilde{Y} \leq \tilde{u}\}} \mid \gamma_{1}, \gamma_{2}, \ldots, \gamma_{n}\right)=\int_{-\infty}^{\tilde{u}} \int_{-\infty}^{\infty} \frac{y}{2 \pi \sigma_{\tilde{Y}} \widehat{\sigma}_{j} \sqrt{1-\rho_{j \tilde{Y}}^{2}}} \times \\
& \times \exp \left(-\frac{1}{2\left(1-\rho_{j \tilde{Y}}^{2}\right)}\left[\frac{x^{2}}{\sigma_{\tilde{Y}}^{2}}-2 \rho_{j \tilde{Y}} \frac{x y}{\sigma_{\tilde{Y}} \widehat{\sigma}_{j}}+\frac{y^{2}}{\widehat{\sigma}_{j}^{2}}\right]\right) d y d x= \\
& =\int_{-\infty}^{\tilde{u}} \frac{x \rho_{j \tilde{Y}} \widehat{\sigma}_{j}}{\sigma_{\tilde{Y}}^{2} \sqrt{2 \pi}} \exp \left(-\frac{x^{2}}{2 \sigma_{\tilde{Y}}^{2}}\right) d x=-\frac{\rho_{j \tilde{Y}} \widehat{\sigma}_{j}}{\sqrt{2 \pi}} \exp \left(-\frac{\tilde{u}^{2}}{2 \sigma_{\tilde{Y}}^{2}}\right),
\end{aligned}
$$

where $\sigma_{\tilde{Y}}, \rho_{j \tilde{Y}}, \widehat{\sigma}_{j}, \tilde{u}$ are defined in $(4.2),(4.3),(4.4),(4.5)$, respectively. Hence

$$
\begin{aligned}
& \mathrm{E}\left(\gamma_{j}^{\zeta}\left(\sum_{l=1}^{n} x_{l} \theta_{l} \gamma_{l}\right)^{\alpha} Y_{j} I_{\left\{\sum_{l=1}^{n} Y_{l} \leq u-\sum_{l=1}^{n} x_{l}\left(r_{l}+\theta_{l} \gamma_{l}\right)\right\}}\right)= \\
= & -\frac{s_{2} x_{j} \sigma_{j}\left(\sum_{l=1}^{n} \kappa_{l} x_{l} \theta_{l}\right)^{\alpha} \kappa_{j}^{\zeta+\frac{1}{2}}}{s_{3} \sqrt{2 \pi}} \mathrm{E}\left(\gamma^{\zeta+\alpha+\frac{1}{2}} \exp \left(-\frac{\left(\widehat{u}-s_{1} \gamma\right)^{2}}{2 s_{3}^{2} \gamma}\right)\right)= \\
= & -\frac{s_{2} x_{j} \sigma_{j}\left(\sum_{l=1}^{n} \kappa_{l} x_{l} \theta_{l}\right)^{\alpha} \exp \left(\frac{\widehat{u} s_{1}^{2}}{s_{3}^{2}}\right) b^{a} \kappa_{j}^{\zeta+\frac{1}{2}}}{s_{3} \Gamma(a) \sqrt{2 \pi}} \times \\
\times & \int_{0}^{\infty} g^{\zeta+\alpha+a-\frac{1}{2}} \exp \left(-\frac{\widehat{u}^{2}}{2 s_{3}^{2} g}-\frac{s_{1}^{2}+2 b s_{3}^{2}}{2 s_{3}^{2}} g\right) d g= \\
= & -\frac{s_{2} x_{j} \sigma_{j}\left(\sum_{l=1}^{n} \kappa_{l} x_{l} \theta_{l}\right)^{\alpha} \exp \left(\frac{\widehat{u} s_{1}}{s_{3}^{2}}\right) b^{a} \kappa_{j}^{\zeta+\frac{1}{2}} \sqrt{2}}{s_{3} \Gamma(a) \sqrt{\pi}} \times \\
\times & \left(\frac{\widehat{u}^{2}}{s_{1}^{2}+2 b s_{3}^{2}}\right)^{\frac{\zeta+\alpha+a}{2}+\frac{1}{4}} \mathrm{~K}_{\zeta+\alpha+a+\frac{1}{2}}\left(\frac{|\widehat{u}| \sqrt{s_{1}^{2}+2 b s_{3}^{2}}}{s_{3}^{2}}\right)
\end{aligned}
$$


due to the formula 3.471.9 from Gradshteyn and Ryzhik [17] when $\widehat{u} \neq 0$. If $\widehat{u}=0$,

$$
\begin{aligned}
& \mathrm{E}\left(\gamma_{j}^{\zeta}\left(\sum_{l=1}^{n} x_{l} \theta_{l} \gamma_{l}\right)^{\alpha} Y_{j} I_{\left\{\sum_{l=1}^{n} Y_{l} \leq u-\sum_{l=1}^{n} x_{l}\left(r_{l}+\theta_{l} \gamma_{l}\right)\right\}}\right)= \\
= & -\frac{s_{2} x_{j} \sigma_{j}\left(\sum_{l=1}^{n} \kappa_{l} x_{l} \theta_{l}\right)^{\alpha} b^{a} \kappa_{j}^{\zeta+\frac{1}{2}}}{s_{3} \Gamma(a) \sqrt{2 \pi}} \int_{0}^{\infty} g^{\zeta+\alpha+a-\frac{1}{2}} \exp \left(-\frac{s_{1}^{2}+2 b s_{3}^{2}}{2 s_{3}^{2}} g\right) d g= \\
= & -\frac{2^{\zeta+\alpha+a} s_{2} s_{3}^{2(\zeta+\alpha+a)} x_{j} \sigma_{j}\left(\sum_{l=1}^{n} \kappa_{l} x_{l} \theta_{l}\right)^{\alpha} b^{a} \Gamma\left(\alpha+a+\frac{1}{2}\right) \kappa_{j}^{\zeta+\frac{1}{2}}}{\Gamma(a)\left(s_{1}^{2}+2 b s_{3}^{2}\right)^{\zeta+\alpha+a+\frac{1}{2}} \sqrt{\pi}} .
\end{aligned}
$$

Thus, we get (3.5) from (4.13) and (4.12).

Proof of Lemma 3.3. Since

$$
\begin{aligned}
& \mathrm{P}\left(\sum_{l=1}^{n} Y_{l} \leq u-\sum_{l=1}^{n} x_{l}\left(r_{l}+\theta_{l} \gamma_{l}\right) \mid \gamma_{1}, \gamma_{2}, \ldots, \gamma_{n}\right)= \\
& =\mathrm{P}\left(\tilde{Y} \leq \tilde{u} \mid \gamma_{1}, \gamma_{2}, \ldots, \gamma_{n}\right)=\mathrm{N}\left(\frac{\tilde{u}}{\sigma_{\tilde{Y}}}\right)
\end{aligned}
$$

we have that

$$
\begin{aligned}
& \mathrm{E}\left(\gamma_{j}^{\zeta}\left(\sum_{l=1}^{n} x_{l} \theta_{l} \gamma_{l}\right)^{\alpha} I_{\left\{\sum_{l=1}^{n} Y_{l} \leq u-\sum_{l=1}^{n} x_{l}\left(r_{l}+\theta_{l} \gamma_{l}\right)\right\}}\right)= \\
& =\kappa_{j}^{\zeta}\left(\sum_{l=1}^{n} x_{l} \theta_{l} \kappa_{l}\right)^{\alpha} \mathrm{E}\left(\gamma^{\zeta+\alpha} \mathrm{N}\left(\frac{\tilde{u}}{\sigma_{\tilde{Y}}}\right)\right)= \\
& =\frac{b^{a} \kappa_{j}^{\zeta}\left(\sum_{l=1}^{n} x_{l} \theta_{l} \kappa_{l}\right)^{\alpha}}{\Gamma(a)} \int_{0}^{\infty} g^{\zeta+\alpha+a-1} \exp (-b g) \mathrm{N}\left(\frac{\widehat{u}-s_{1} g}{s_{3} \sqrt{g}}\right) d g
\end{aligned}
$$

Hence we get similarly to the proof of Lemma 3.1 that

$$
\begin{aligned}
& \mathrm{E}\left(\gamma_{j}^{\zeta}\left(\sum_{l=1}^{n} x_{l} \theta_{l} \gamma_{l}\right)^{\alpha} I_{\left\{\sum_{l=1}^{n} Y_{l} \leq u-\sum_{l=1}^{n} x_{l}\left(r_{l}+\theta_{l} \gamma_{l}\right)\right\}}\right)= \\
& =\frac{b^{a} \kappa_{j}^{\zeta}\left(\sum_{l=1}^{n} x_{l} \theta_{l} \kappa_{l}\right)^{\alpha} \Gamma\left(\zeta+\alpha+a+\frac{1}{2}\right)}{\Gamma(a) b^{\zeta+\alpha+a} \sqrt{2 \pi}} \times \\
& \left(\frac{\mathrm{B}\left(\frac{1}{2}, \zeta+\alpha+a\right)}{\sqrt{2}}-\frac{s_{1}}{s_{3} \sqrt{b}} \mathrm{~F}\left(\zeta+\alpha+a+\frac{1}{2}, \frac{1}{2}, \frac{3}{2} ;-\frac{s_{1}^{2}}{2 b s_{3}^{2}}\right)\right)
\end{aligned}
$$


if $\widehat{u}=0$ and

$$
\begin{aligned}
& \mathrm{E}\left(\gamma_{j}^{\zeta}\left(\sum_{l=1}^{n} x_{l} \theta_{l} \gamma_{l}\right)^{\alpha} I_{\left\{\sum_{l=1}^{n} Y_{l} \leq u-\sum_{l=1}^{n} x_{l}\left(r_{l}+\theta_{l} \gamma_{l}\right)\right\}}\right)= \\
& =\frac{b^{a} \kappa_{j}^{\zeta}\left(\sum_{l=1}^{n} x_{l} \theta_{l} \kappa_{l}\right)^{\alpha}|s|^{\zeta+\alpha+a-\frac{1}{2}} e^{s}(1+q)^{\zeta+\alpha+a}}{\Gamma(a) b^{\zeta+\alpha+a} \sqrt{2 \pi}} \times \\
& \left(\mathrm{B}(\zeta+\alpha+a, 1)\left(|s| \mathrm{K}_{\zeta+\alpha+a+\frac{1}{2}}(|s|)+s \mathrm{~K}_{\zeta+\alpha+a-\frac{1}{2}}(|s|)\right) \times\right. \\
& \Phi\left(\zeta+\alpha+a, 1-\zeta-\alpha-a, \zeta+\alpha+a+1 ; \frac{1+q}{2},-s(1+q)\right)- \\
& -(1+q) s \mathrm{~B}(\zeta+\alpha+a+1,1) \mathrm{K}_{\zeta+\alpha+a-\frac{1}{2}}(|s|) \times \\
& \left.\Phi\left(\zeta+\alpha+a+1,1-\zeta-\alpha-a, \zeta+\alpha+a+2 ; \frac{1+q}{2},-s(1+q)\right)\right)
\end{aligned}
$$

when $\widehat{u} \neq 0$. We have (3.6) from (4.14) and (4.15).

Proof of Lemma 3.4. We have with respect to (4.1) that

$$
\begin{aligned}
& \mathrm{E}\left(\gamma_{j}^{\zeta}\left(\sum_{l=1}^{n} x_{l} \theta_{l} \gamma_{l}\right)^{\alpha} \sum_{l=1}^{n} Y_{l} I_{\left\{\sum_{l=1}^{n} Y_{l} \leq u-\sum_{l=1}^{n} x_{l}\left(r_{l}+\theta_{l} \gamma_{l}\right)\right\}} \mid \gamma_{1}, \gamma_{2}, \ldots, \gamma_{n}\right)= \\
& =\gamma_{j}^{\zeta}\left(\sum_{l=1}^{n} x_{l} \theta_{l} \gamma_{l}\right)^{\alpha} \mathrm{E}\left(\tilde{Y} I_{\left\{\tilde{Y} \leq u-\sum_{l=1}^{n} x_{l}\left(r_{l}+\theta_{l} \gamma_{l}\right)\right\}} \mid \gamma_{1}, \gamma_{2}, \ldots, \gamma_{n}\right)= \\
& =\gamma_{j}^{\zeta}\left(\sum_{l=1}^{n} x_{l} \theta_{l} \gamma_{l}\right)^{\alpha} \int_{-\infty}^{\tilde{u}} \frac{x}{\sigma_{\tilde{Y}} \sqrt{2 \pi}} \exp \left(-\frac{x^{2}}{2 \sigma_{\tilde{Y}}^{2}}\right) d x= \\
& =-\frac{\sigma_{\tilde{Y}} \gamma_{j}^{\zeta}\left(\sum_{l=1}^{n} x_{l} \theta_{l} \gamma_{l}\right)^{\alpha}}{\sqrt{2 \pi}} \exp \left(-\frac{\tilde{u}^{2}}{2 \sigma_{\tilde{Y}}^{2}}\right) .
\end{aligned}
$$

Hence

$$
\begin{aligned}
& \mathrm{E}\left(\gamma_{j}^{\zeta}\left(\sum_{l=1}^{n} x_{l} \theta_{l} \gamma_{l}\right)^{\alpha} \sum_{l=1}^{n} Y_{l} I_{\left\{\sum_{l=1}^{n} Y_{l} \leq u-\sum_{l=1}^{n} x_{l}\left(r_{l}+\theta_{l} \gamma_{l}\right)\right\}}\right)= \\
& =-\frac{s_{3} b^{a} s_{1}^{\alpha} \kappa_{j}^{\zeta}}{\Gamma(a) \sqrt{2 \pi}} \int_{0}^{\infty} g^{\zeta+\alpha+a-\frac{1}{2}} \exp \left(-b g-\frac{\left(\widehat{u}-s_{1} g\right)^{2}}{2 s_{3}^{2} g}\right) d g= \\
& =-\frac{s_{3} b^{a} s_{1}^{\alpha} \kappa_{j}^{\zeta} \exp \left(\frac{\widehat{u} s_{1}}{s_{3}^{2}}\right) \sqrt{2}}{\Gamma(a) \sqrt{\pi}}\left(\frac{\widehat{u}^{2}}{s_{1}^{2}+2 b s_{3}^{2}}\right)^{\frac{\zeta+\alpha+a}{2}+\frac{1}{4}} \times \\
& \times \mathrm{K}_{\zeta+\alpha+a+\frac{1}{2}}\left(\frac{|\widehat{u}| \sqrt{s_{1}^{2}+2 b s_{3}^{2}}}{s_{3}^{2}}\right)
\end{aligned}
$$


if $\widehat{u} \neq 0$ and

$$
\begin{aligned}
& \mathrm{E}\left(\gamma_{j}^{\zeta}\left(\sum_{l=1}^{n} x_{l} \theta_{l} \gamma_{l}\right)^{\alpha} \sum_{l=1}^{n} Y_{l} I_{\left\{\sum_{l=1}^{n} Y_{l} \leq u-\sum_{l=1}^{n} x_{l}\left(r_{l}+\theta_{l} \gamma_{l}\right)\right\}}\right)= \\
& =-\frac{s_{3} b^{a} s_{1}^{\alpha} \kappa_{j}^{\zeta}}{\Gamma(a) \sqrt{2 \pi}} \Gamma\left(\zeta+\alpha+a+\frac{1}{2}\right)\left(\frac{2 s_{3}^{2}}{s_{1}^{2}+2 b s_{3}^{2}}\right)^{\zeta+\alpha+a+\frac{1}{2}}
\end{aligned}
$$

when $\widehat{u}=0$ similarly to (4.8) and (4.9), respectively. We get (3.7) from (4.16) and (4.17).

Proof of Lemma 3.5. Conditional expectation

$$
\begin{aligned}
& \mathrm{E}\left(\left(\sum_{l=1}^{n} Y_{l}\right)^{2} I_{\left\{\sum_{l=1}^{n} Y_{l} \leq u-\sum_{l=1}^{n} x_{l}\left(r_{l}+\theta_{l} \gamma_{l}\right)\right\}} \mid \gamma_{1}, \gamma_{2}, \ldots, \gamma_{n}\right)= \\
& \left.=\mathrm{E}\left(\tilde{Y}^{2} I_{\left\{\tilde{Y} \leq u-\sum_{l=1}^{n} x_{l}\left(r_{l}+\theta_{l} \gamma_{l}\right)\right\}}\right) \mid \gamma_{1}, \gamma_{2}, \ldots, \gamma_{n}\right)= \\
& =\int_{-\infty}^{\tilde{u}} \frac{x^{2}}{\sigma_{\tilde{Y}} \sqrt{2 \pi}} \exp \left(-\frac{x^{2}}{2 \sigma_{\tilde{Y}}^{2}}\right) d x=-\frac{\sigma_{\tilde{Y}}}{\sqrt{2 \pi}}\left(\tilde{u} \exp \left(-\frac{\tilde{u}^{2}}{2 \sigma_{\tilde{Y}}^{2}}\right)-\right. \\
& \left.-\int_{-\infty}^{\tilde{u}} \exp \left(-\frac{x^{2}}{2 \sigma_{\tilde{Y}}^{2}}\right) d x\right)=-\frac{\tilde{u} \sigma_{\tilde{Y}}}{\sqrt{2 \pi}} \exp \left(-\frac{\tilde{u}^{2}}{2 \sigma_{\tilde{Y}}^{2}}\right)+\sigma_{\tilde{Y}}^{2} \mathrm{~N}\left(\frac{\tilde{u}}{\sigma_{\tilde{Y}}}\right) .
\end{aligned}
$$

Therefore

$$
\begin{aligned}
& \mathrm{E}\left(\left(\sum_{l=1}^{n} Y_{l}\right)^{2} I_{\left\{\sum_{l=1}^{n} Y_{l} \leq u-\sum_{l=1}^{n} x_{l}\left(r_{l}+\theta_{l} \gamma_{l}\right)\right\}}\right)= \\
= & s_{3}\left(\frac{s_{1}}{\sqrt{2 \pi}} \mathrm{E}\left(\gamma^{\frac{3}{2}} \exp \left(-\frac{s_{1}^{2}}{2 s_{3}^{2}} \gamma\right)\right)+s_{3} \mathrm{E}\left(\gamma \mathrm{N}\left(-\frac{s_{1}}{s_{3}} \sqrt{\gamma}\right)\right)\right)= \\
= & \frac{s_{3} b^{a}}{\Gamma(a)}\left(\frac{s_{1}}{\sqrt{2 \pi}} \int_{0}^{\infty} g^{a+\frac{1}{2}} \exp \left(-\frac{s_{1}^{2}+2 b s_{3}^{2}}{2 s_{3}^{2}} g\right) d g+\right. \\
+ & \left.s_{3} \int_{0}^{\infty} g^{a} \mathrm{~N}\left(-\frac{s_{1}}{s_{3}} \sqrt{g}\right) \exp (-b g) d g\right)= \\
= & \frac{s_{3} b^{a}}{\Gamma(a)}\left(\frac{s_{1}}{\sqrt{2 \pi}} \Gamma\left(a+\frac{3}{2}\right)\left(\frac{2 s_{3}^{2}}{s_{1}^{2}+2 b s_{3}^{2}}\right)^{a+\frac{3}{2}}+\right. \\
+ & \left.\frac{s_{3} \Gamma\left(a+\frac{3}{2}\right)}{b^{a+1} \sqrt{2 \pi}}\left[\frac{\mathrm{B}\left(\frac{1}{2}, a+1\right)}{\sqrt{2}}-\frac{s_{1}}{s_{3} \sqrt{b}} \mathrm{~F}\left(a+\frac{3}{2}, \frac{1}{2}, \frac{3}{2} ;-\frac{s_{1}^{2}}{2 b s_{3}^{2}}\right)\right]\right)
\end{aligned}
$$


if $\widehat{u}=0$ as in (4.9) and (4.10). When $\widehat{u} \neq 0$,

$$
\begin{aligned}
& \mathrm{E}\left(\left(\sum_{l=1}^{n} Y_{l}\right)^{2} I_{\left\{\sum_{l=1}^{n} Y_{l} \leq u-\sum_{l=1}^{n} x_{l}\left(r_{l}+\theta_{l} \gamma_{l}\right)\right\}}\right)=s_{3}\left(s_{3} \mathrm{E}\left(\gamma \mathrm{N}\left(\frac{\widehat{u}-s_{1} \gamma}{s_{3} \sqrt{\gamma}}\right)\right)+\right. \\
& \left.+\frac{1}{\sqrt{2 \pi}}\left[s_{1} \mathrm{E}\left(\gamma^{\frac{3}{2}} \exp \left(-\frac{\left(\widehat{u}-s_{1} \gamma\right)^{2}}{2 s_{3}^{2} \gamma}\right)\right)-\widehat{u} \mathrm{E}\left(\gamma^{\frac{1}{2}} \exp \left(-\frac{\left(\widehat{u}-s_{1} \gamma\right)^{2}}{2 s_{3}^{2} \gamma}\right)\right)\right]\right)= \\
& =\frac{s_{3} b^{a}}{\Gamma(a)}\left(s_{3} \int_{0}^{\infty} g^{a} \mathrm{~N}\left(\frac{\widehat{u}-s_{1} g}{s_{3} \sqrt{g}}\right) \exp (-b g) d g+\right. \\
& +\frac{\exp \left(\frac{\widehat{u} s_{1}}{s_{3}^{2}}\right)}{\sqrt{2 \pi}}\left[s_{1} \int_{0}^{\infty} g^{a+\frac{1}{2}} \exp \left(-\frac{\widehat{u}^{2}}{2 s_{3}^{2} g}-\frac{s_{1}^{2}+2 b s_{3}^{2}}{2 s_{3}^{2}} g\right) d g-\right. \\
& \left.\left.-\widehat{u} \int_{0}^{\infty} g^{a-\frac{1}{2}} \exp \left(-\frac{\widehat{u}^{2}}{2 s_{3}^{2} g}-\frac{s_{1}^{2}+2 b s_{3}^{2}}{2 s_{3}^{2}} g\right) d g\right]\right)
\end{aligned}
$$

and hence

$$
\begin{aligned}
& \mathrm{E}\left(\left(\sum_{l=1}^{n} Y_{l}\right)^{2} I_{\left\{\sum_{l=1}^{n} Y_{l} \leq u-\sum_{l=1}^{n} x_{l}\left(r_{l}+\theta_{l} \gamma_{l}\right)\right\}}\right)= \\
& =\frac{s_{3} b^{a}}{\Gamma(a)}\left(\frac { s _ { 3 } | s | ^ { a + \frac { 1 } { 2 } } e ^ { s } ( 1 + q ) ^ { a + 1 } } { b ^ { a + 1 } \sqrt { 2 \pi } } \left[\mathrm { B } ( a + 1 , 1 ) \left(|s| \mathrm{K}_{a+\frac{3}{2}}(|s|)+\right.\right.\right. \\
& \left.+s \mathrm{~K}_{a+\frac{1}{2}}(|s|)\right) \Phi\left(a+1,-a, a+2 ; \frac{1+q}{2},-s(1+q)\right)- \\
& \left.(1+q) s \mathrm{~B}(a+2,1) \mathrm{K}_{a+\frac{1}{2}}(|s|) \Phi\left(a+2,-a, a+3 ; \frac{1+q}{2},-s(1+q)\right)\right] \\
& +\frac{\exp \left(\frac{\widehat{u} s_{1}}{s_{3}^{2}}\right) \sqrt{2}}{\sqrt{\pi}}\left[s_{1}\left(\frac{\widehat{u}^{2}}{s_{1}^{2}+2 b s_{3}^{2}}\right)^{\frac{a}{2}+\frac{3}{4}} \mathrm{~K}_{a+\frac{3}{2}}\left(\frac{|\widehat{u}| \sqrt{s_{1}^{2}+2 b s_{3}^{2}}}{s_{3}^{2}}\right)-\right. \\
& \left.\left.-\widehat{u}\left(\frac{\widehat{u}^{2}}{s_{1}^{2}+2 b s_{3}^{2}}\right)^{\frac{a}{2}+\frac{1}{4}} \mathrm{~K}_{a+\frac{1}{2}}\left(\frac{|\widehat{u}| \sqrt{s_{1}^{2}+2 b s_{3}^{2}}}{s_{3}^{2}}\right)\right]\right)
\end{aligned}
$$

in this case similarly to (4.8) and (4.11). We establish (3.8) from (4.18) and (4.19).

Proof of Theorem 3.1. We have that

$$
\begin{aligned}
& \beta^{-}=\frac{\mathrm{E}\left[\left(X_{j}-\mathrm{E} X_{j}\right)(X-\mathrm{E} X) I_{\{X \leq u\}}\right]}{\mathrm{E}\left[(X-\mathrm{E} X)^{2} I_{\{X \leq u\}}\right]}= \\
= & \frac{\mathrm{E}\left(X_{j} X I_{\{X \leq u\}}\right)-\mathrm{E} X_{j} \mathrm{E}\left(X I_{\{X \leq u\}}\right)}{\mathrm{E}\left(X^{2} I_{\{X \leq u\}}\right)-2 \mathrm{E} X \mathrm{E}\left(X I_{\{X \leq u\}}\right)+(\mathrm{E} X)^{2} \mathrm{P}(X \leq u)}+ \\
= & \frac{\mathrm{E} X_{j} \mathrm{E} X \mathrm{P}(X \leq u)-\mathrm{E} X \mathrm{E}\left(X_{j} I_{\{X \leq u\}}\right)}{\mathrm{E}\left(X^{2} I_{\{X \leq u\}}\right)-2 \mathrm{E} X \mathrm{E}\left(X I_{\{X \leq u\}}\right)+(\mathrm{E} X)^{2} \mathrm{P}(X \leq u)}
\end{aligned}
$$

and hence it is needed to compute consequently $\mathrm{E}\left(X_{j} X I_{\{X \leq u\}}\right), \mathrm{E}\left(X I_{\{X \leq u\}}\right), \mathrm{E}\left(X_{j} I_{\{X \leq u\}}\right), \mathrm{P}(X \leq u)$ and $\mathrm{E}\left(X^{2} I_{\{X \leq u\}}\right)$. 
One can see that

$$
\begin{aligned}
& \mathrm{E}\left(X_{j} X I_{\{X \leq u\}} \mid \gamma_{1}, \gamma_{2}, \ldots, \gamma_{n}\right)=x_{j}\left(r_{j}+\theta_{j} \gamma_{j}\right) \sum_{l=1}^{n} x_{l}\left(r_{l}+\theta_{l} \gamma_{l}\right) \times \\
& \times \mathrm{P}\left(\sum_{l=1}^{n} Y_{l} \leq u-\sum_{l=1}^{n} x_{l}\left(r_{l}+\theta_{l} \gamma_{l}\right) \mid \gamma_{1}, \gamma_{2}, \ldots, \gamma_{n}\right)+x_{j}\left(r_{j}+\theta_{j} \gamma_{j}\right) \times \\
& \times \mathrm{E}\left(\sum_{l=1}^{n} Y_{l} I_{\left\{\sum_{l=1}^{n} Y_{l} \leq u-\sum_{l=1}^{n} x_{l}\left(r_{l}+\theta_{l} \gamma_{l}\right)\right\}} \mid \gamma_{1}, \gamma_{2}, \ldots, \gamma_{n}\right)+\sum_{l=1}^{n} x_{l}\left(r_{l}+\theta_{l} \gamma_{l}\right) \times \\
& \times \mathrm{E}\left(Y_{j} I_{\left\{\sum_{l=1}^{n} Y_{l} \leq u-\sum_{l=1}^{n} x_{l}\left(r_{l}+\theta_{l} \gamma_{l}\right)\right\}} \mid \gamma_{1}, \gamma_{2}, \ldots, \gamma_{n}\right)+ \\
& +\mathrm{E}\left(Y_{j} \sum_{l=1}^{n} Y_{l} I_{\left\{\sum_{l=1}^{n} Y_{l} \leq u-\sum_{l=1}^{n} x_{l}\left(r_{l}+\theta_{l} \gamma_{l}\right)\right\}} \mid \gamma_{1}, \gamma_{2}, \ldots, \gamma_{n}\right) \text {. }
\end{aligned}
$$

Hence we have that

$$
\begin{aligned}
& \mathrm{E}\left(X_{j} X I_{\{X \leq u\}}\right)=x_{j}\left(r_{j}\left[\widehat{s} f_{3}(0,0)+f_{3}(0,1)\right]+\right. \\
& \left.+\theta_{j}\left[\widehat{s} f_{3}(1,0)+f_{3}(1,1)\right]+r_{j} f_{4}(0,0)+\theta_{j} f_{4}(1,0)\right)+ \\
& +\widehat{s} f_{2}(0,0)+f_{2}(0,1)+f_{1} .
\end{aligned}
$$

Next,

$$
\begin{aligned}
& \mathrm{E}\left(X I_{\{X \leq u\}} \mid \gamma_{1}, \gamma_{2}, \ldots, \gamma_{n}\right)=\sum_{l=1}^{n} x_{l}\left(r_{l}+\theta_{l} \gamma_{l}\right) \times \\
\times & \mathrm{P}\left(\sum_{l=1}^{n} Y_{l} \leq u-\sum_{l=1}^{n} x_{l}\left(r_{l}+\theta_{l} \gamma_{l}\right) \mid \gamma_{1}, \gamma_{2}, \ldots, \gamma_{n}\right)+ \\
+ & \mathrm{E}\left(\sum_{l=1}^{n} Y_{l} I_{\left\{\sum_{l=1}^{n} Y_{l} \leq u-\sum_{l=1}^{n} x_{l}\left(r_{l}+\theta_{l} \gamma_{l}\right)\right\}} \mid \gamma_{1}, \gamma_{2}, \ldots, \gamma_{n}\right)
\end{aligned}
$$

and therefore

$$
\mathrm{E}\left(X I_{\{X \leq u\}}\right)=\widehat{s} f_{3}(0,0)+f_{3}(0,1)+f_{4}(0,0) .
$$

Further,

$$
\begin{aligned}
& \mathrm{E}\left(X_{j} I_{\{X \leq u\}} \mid \gamma_{1}, \gamma_{2}, \ldots, \gamma_{n}\right)=x_{j}\left(r_{j}+\theta_{j} \gamma_{j}\right) \times \\
\times & \mathrm{P}\left(\sum_{l=1}^{n} Y_{l} \leq u-\sum_{l=1}^{n} x_{l}\left(r_{l}+\theta_{l} \gamma_{l}\right) \mid \gamma_{1}, \gamma_{2}, \ldots, \gamma_{n}\right)+ \\
+ & \mathrm{E}\left(Y_{j} I_{\left\{\sum_{l=1}^{n} Y_{l} \leq u-\sum_{l=1}^{n} x_{l}\left(r_{l}+\theta_{l} \gamma_{l}\right)\right\} \mid} \mid \gamma_{1}, \gamma_{2}, \ldots, \gamma_{n}\right)
\end{aligned}
$$

and then

$$
\mathrm{E}\left(X_{j} I_{\{X \leq u\}}\right)=x_{j}\left(r_{j} f_{3}(0,0)+\theta_{j} f_{3}(1,0)\right)+f_{2}(0,0)
$$


Also,

$$
\mathrm{P}\left(X \leq u \mid \gamma_{1}, \gamma_{2}, \ldots, \gamma_{n}\right)=\mathrm{P}\left(\sum_{l=1}^{n} Y_{l} \leq u-\sum_{l=1}^{n} x_{l}\left(r_{l}+\theta_{l} \gamma_{l}\right) \mid \gamma_{1}, \gamma_{2}, \ldots, \gamma_{n}\right)
$$

and hence

$$
\mathrm{P}(X \leq u)=f_{3}(0,0) .
$$

Moreover,

$$
\begin{aligned}
& \mathrm{E}\left(X^{2} I_{\{X \leq u\}} \mid \gamma_{1}, \gamma_{2}, \ldots, \gamma_{n}\right)=\left(\sum_{l=1}^{n} x_{l}\left(r_{l}+\theta_{l} \gamma_{l}\right)\right)^{2} \times \\
& \times \mathrm{P}\left(\sum_{l=1}^{n} Y_{l} \leq u-\sum_{l=1}^{n} x_{l}\left(r_{l}+\theta_{l} \gamma_{l}\right) \mid \gamma_{1}, \gamma_{2}, \ldots, \gamma_{n}\right)+ \\
& +2 \sum_{l=1}^{n} x_{l}\left(r_{l}+\theta_{l} \gamma_{l}\right) \mathrm{E}\left(\sum_{l=1}^{n} Y_{l} I_{\left\{\sum_{l=1}^{n} Y_{l} \leq u-\sum_{l=1}^{n} x_{l}\left(r_{l}+\theta_{l} \gamma_{l}\right)\right\}} \mid \gamma_{1}, \gamma_{2}, \ldots, \gamma_{n}\right)+ \\
& +\mathrm{E}\left(\left(\sum_{l=1}^{n} Y_{l}\right)^{2} I_{\left\{\sum_{l=1}^{n} Y_{l} \leq u-\sum_{l=1}^{n} x_{l}\left(r_{l}+\theta_{l} \gamma_{l}\right)\right\}} \mid \gamma_{1}, \gamma_{2}, \ldots, \gamma_{n}\right)
\end{aligned}
$$

and

$$
\begin{aligned}
& \mathrm{E}\left(X^{2} I_{\{X \leq u\}}\right)=\widehat{s}^{2} f_{3}(0,0)+2 \widehat{s} f_{3}(0,1)+ \\
& +f_{3}(0,2)+2 \widehat{s} f_{4}(0,0)+2 f_{4}(0,1)+f_{5}(0,0)
\end{aligned}
$$

Keeping in mind the identities

$$
\mathrm{E} X_{j}=h_{j} \quad \text { and } \quad \mathrm{E} X=h,
$$

we get exploiting (4.20)-(4.25) that

$$
\beta^{-}=\frac{\beta_{n}^{-}}{\beta_{d}^{-}}
$$

where

$$
\begin{aligned}
& \beta_{n}^{-}=x_{j}\left(r_{j}\left[\widehat{s} f_{3}(0,0)+f_{3}(0,1)\right]+\right. \\
& \left.+\theta_{j}\left[\widehat{s} f_{3}(1,0)+f_{3}(1,1)\right]+r_{j} f_{4}(0,0)+\theta_{j} f_{4}(1,0)\right)+ \\
& +\widehat{s} f_{2}(0,0)+f_{2}(0,1)+f_{1}-h_{j}\left[\widehat{s} f_{3}(0,0)+\right. \\
& \left.f_{3}(0,1)+f_{4}(0,0)\right]+h_{j} h f_{3}(0,0)-h\left[x_{j}\left(r_{j} f_{3}(0,0)+\theta_{j} f_{3}(1,0)\right)+f_{2}(0,0)\right]
\end{aligned}
$$


and

$$
\begin{aligned}
& \beta_{d}^{-}=\widehat{s}^{2} f_{3}(0,0)+2 \widehat{s} f_{3}(0,1)+ \\
& +f_{3}(0,2)+2 \widehat{s} f_{4}(0,0)+2 f_{4}(0,1)+f_{5}(0,0)- \\
& -2 h\left[\widehat{s} f_{3}(0,0)+f_{3}(0,1)+f_{4}(0,0)\right]+h^{2} f_{3}(0,0) .
\end{aligned}
$$

Proof of Theorem 3.2. One can observe that

$$
\begin{aligned}
& \beta^{+}=\frac{\mathrm{E}\left[\left(X_{j}-\mathrm{E} X_{j}\right)(X-\mathrm{E} X) I_{\{X \geq u\}}\right]}{\mathrm{E}\left[(X-\mathrm{E} X)^{2} I_{\{X \geq u\}}\right]}= \\
= & \frac{\mathrm{E}\left[\left(X_{j}-\mathrm{E} X_{j}\right)(X-\mathrm{E} X)\right]-\mathrm{E}\left[\left(X_{j}-\mathrm{E} X_{j}\right)(X-\mathrm{E} X) I_{\{X \leq u\}}\right]}{\mathrm{E}\left[(X-\mathrm{E} X)^{2}\right]-\mathrm{E}\left[(X-\mathrm{E} X)^{2} I_{\{X \leq u\}}\right]}= \\
= & \frac{\mathrm{E} X_{j} X-\mathrm{E} X_{j} \mathrm{E} X-\mathrm{E}\left[\left(X_{j}-\mathrm{E} X_{j}\right)(X-\mathrm{E} X) I_{\{X \leq u\}}\right]}{\mathrm{E} X^{2}-(\mathrm{E} X)^{2}-\mathrm{E}\left[(X-\mathrm{E} X)^{2} I_{\{X \leq u\}}\right]} .
\end{aligned}
$$

Since

$$
\begin{aligned}
& \mathrm{E} X_{j} X=\mathrm{E}\left[x_{j}\left(r_{j}+\theta_{j} \gamma_{j}+\sigma_{j} \sqrt{\gamma_{j}} N_{j}\right) \sum_{l=1}^{n} x_{l}\left(r_{l}+\theta_{l} \gamma_{l}+\sigma_{l} \sqrt{\gamma_{l}} N_{l}\right)\right]= \\
& =x_{j}\left[r_{j} \sum_{l=1}^{n} x_{l}\left(r_{l}+\frac{\theta_{l} a_{l}}{b_{l}}\right)+\theta_{j} \sum_{l=1}^{n} x_{l}\left(\frac{r_{l} a_{j}}{b_{j}}+\theta_{l} \mathrm{E} \gamma_{j} \gamma_{l}\right)+\right. \\
& \left.+\sigma_{j} \sum_{l=1}^{n} x_{l}\left(r_{l}+\sigma_{l} \mathrm{E} \sqrt{\gamma_{l} \gamma_{j}} N_{l} N_{j}\right)\right]=x_{j}\left[r_{j} \sum_{l=1}^{n} x_{l}\left(r_{l}+\frac{\theta_{l} a_{l}}{b_{l}}\right)+\right. \\
& \left.+\theta_{j} \sum_{l=1}^{n} x_{l}\left(\frac{r_{l} a_{j}}{b_{j}}+\frac{\theta_{l} \kappa_{l} \kappa_{j}(a+1) a}{b^{2}}\right)+\sigma_{j} \sum_{l=1}^{n} x_{l}\left(r_{l}+\frac{\sigma_{l} \rho_{l j} a \sqrt{\kappa_{l} \kappa_{j}}}{b}\right)\right]=\widehat{h}_{j}
\end{aligned}
$$

and

$$
\begin{aligned}
& \mathrm{E} X^{2}=\mathrm{E}\left(\sum_{l=1}^{n} x_{l}\left(r_{l}+\theta_{l} \gamma_{l}+\sigma_{l} \sqrt{\gamma_{l}} N_{l}\right)\right)^{2}= \\
= & \mathrm{E}\left(\sum_{l, m=1}^{n} x_{l} x_{m}\left(r_{l}+\theta_{l} \gamma_{l}+\sigma_{l} \sqrt{\gamma_{l}} N_{l}\right)\left(r_{m}+\theta_{m} \gamma_{m}+\sigma_{m} \sqrt{\gamma_{m}} N_{m}\right)\right)= \\
= & \sum_{l, m=1}^{n} x_{l} x_{m}\left(r_{l}\left(r_{m}+\frac{\theta_{m} a_{m}}{b_{m}}\right)+\theta_{l}\left(\frac{r_{m} a_{l}}{b_{l}}+\frac{\theta_{m} \kappa_{l} \kappa_{m} a(a+1)}{b^{2}}\right)+\right. \\
+ & \left.\frac{\sigma_{l} \sigma_{m} \rho_{l m} a \sqrt{\kappa_{l} \kappa_{m}}}{b}\right)=\widehat{h},
\end{aligned}
$$

we get that

$$
\beta^{+}=\frac{\widehat{h}_{j}-h_{j} h-\beta_{n}^{-}}{\widehat{h}-h^{2}-\beta_{d}^{-}} .
$$

Conflicts of Interest: The author(s) declare that there are no conflicts of interest regarding the publication of this paper. 


\section{ReFERENCES}

[1] Y. Altigan, T.G. Bali, K.O. Demirtas and A.D. Gunaydin, Downside Beta and Equity Returns around the World, J. Portfolio Manage. 44 (7) (2018), 39-54.

[2] A. Ang, J. Chen and Y. Xing, Downside risk, Rev. Financ. Stud. 19 (4) (2006), 1191-1239.

[3] K. Ano and R.V. Ivanov, On exact pricing of FX options in multivariate time-changed Lévy models, Rev. Deriv. Res. 19(3) (2016), 201-216.

[4] U. Ayub, S. Kausar, U. Noreen, M. Zakaria and I. Abbas Jadoon, Downside Risk-Based Six-Factor Capital Asset Pricing Model (CAPM): A New Paradigm in Asset Pricing, Sustainability 12 (2020), 6756.

[5] H. Bateman and A. Erdélyi, Higher Transcendental Functions, McGraw-Hill, New York, 1953.

[6] J. Berkowitz, M. Pritsker, M. Gibson and H. Zhou, How accurate are value-at-risk models at commercial banks, J. Finance, 57 (2002), 1093-1111.

[7] A.M. Chaudhry, A. Qadir, H.M. Srivastava and R.B. Paris, Extended Hypergeometric and Confluent Hypergeometric Functions, Appl. Math. Comput. 159(2) (2004), 589-602.

[8] S.X. Chen and C.Y. Tang, Nonparametric inference of value-at-risk for dependent financial returns, J. Financ. Econ. 3(2) (2005), 227-255.

[9] R. Cont, R. Deguest and X.D. He, Loss-based risk measures, Stat. Risk Model. 30(2) (2013), 133-167.

[10] R. Cont and J. Sirignano, Universal features of price formation in financial markets: perspectives from deep learning, Quant. Finance, 19(9) (2019), 1449-1459.

[11] R. Cont and L. Wagalath, Institutional investors and the dependence structure of asset returns, Int. J. Theor. Appl. Finance, 19(2) (2016), 1650010.

[12] E.A. Daal and D.B. Madan, An Empirical Examination of the Variance-Gamma Model for Foreign Currency Options, J. Bus. 78(6) (2005), 2121-2152.

[13] J. Estrada, Mean-semivariance behavior: Downside risk and capital asset pricing, Int. Rev. Econ. Finance, 16(2) (2007), $169-185$.

[14] R. Finlay and E. Seneta, Stationary-increment student and variance-gamma processes, J. Appl. Probab. 43 (2006), $441-453$.

[15] M. Flora and T. Vargiolu, Price dynamics in the European Union Emissions Trading System and evaluation of its ability to boost emission-related investment decisions, Eur. J. Oper. Res. 280 (2020), 383-394.

[16] A. Göncü, M.O. Karahan and T.U. Kuzubas, A comparative goodness-of-fit analysis of distributions of some Lévy processes and Heston model to stock index returns, North Amer. J. Econ. Finance, 36 (2016), 69-83.

[17] I.S. Gradshteyn and I.M. Ryzhik, Table of Integrals, Series and Products, Academic Press, New York, 1980.

[18] A. Guy, Upside and Downside Beta Portfolio Construction: A Different Approach to Risk Measurement and Portfolio Construction, Risk Gov. Control: Financ. Mark. Inst. 5(4) (2015), 243-251.

[19] W. Hogan and J. Warren, Computation of the efficient boundary in the ES portfolio selection model, J. Financ. Quant. Anal. 7(4) (1972), 1881-1896.

[20] R.V. Ivanov, On risk measuring in the variance-gamma model, Stat. Risk Model. 35(1-2) (2018), 23-33.

[21] R.V. Ivanov, A credit-risk valuation under the variance-gamma asset return, Risks, 6(2) (2018), 58.

[22] K. Kalinchenko, S. Uryasev and R.T. Rockafellar, Calibrating Risk Preferences with Generalized CAPM Based on Mixed CVaR Deviation, J. Risk, 15(1) (2012), 45-70.

[23] D. Linders and B. Stassen, The multivariate variance gamma model: basket option pricing and calibration, Quant. Finance, 16(4) (2016), 555-572. 
[24] E. Luciano, M. Marena and P. Semeraro, Dependence calibration and portfolio fit with factor-based subordinators, Quant. Finance, 16(7) (2016), 1037-1052.

[25] E. Luciano and W. Schoutens, A multivariate jump-driven financial asset model, Quant. Finance, 6(5) (2016), 385-402.

[26] D. Madan, P. Carr and E. Chang, The Variance Gamma Process and Option Pricing. Eur. Finance Rev. 2 (1998), 79-105.

[27] D. Madan and F. Milne, Option pricing with VG martingale components, Math. Finance, 1(4) (1991), 39-55.

[28] D. Madan and E. Seneta, The Variance Gamma (V.G.) Model for Share Market Returns, J. Bus. 63 (1990), 511-524.

[29] A. Mafusalov and S. Uryasev, CVaR (Superquantile) Norm: Stochastic Case, Europ. J. Oper. Res. 249 (2016), $200-208$.

[30] H. Markowitz, Portfolio selection: Efficient diversification of investments, Yale University Press, Yale, 1959.

[31] T. Moosbrucker, Explaining the correlation smile using variance gamma distributions, J. Fixed Income, 16(1) (2006), $71-87$.

[32] S. Mozumder, G. Sorwar and K. Dowd, Revisiting variance gamma pricing: An application to s\&p500 index options, Int. J. Financ. Eng. 2(2) (2015), 1550022.

[33] S.T. My, Credit risk and bank stability of Vietnam commercial bank: a BK approach, Int. J. Anal. Appl. 18(6) (2020), $1066-1082$.

[34] T. Nitithumbundit and J.S.K. Chan, ECM Algorithm for Auto-Regressive Multivariate Skewed Variance Gamma Model with Unbounded Density, Methodol. Comput. Appl. Probab. 22 (2020), 1169-1191.

[35] T. Post and P. Van Vliet, Downside risk and asset pricing, J. Bank. Finance, 30(3) (2006), 823-849.

[36] A. Rathgeber, J. Stadler and S. Stöck, Modeling share returns - an empirical study on the Variance Gamma model, J. Econ. Finance, 40(4) (2016), 653-682.

[37] R.T. Rockafellar and S. Uryasev, Optimization of conditional value-at-risk, J. Risk, 2 (2000), 21-41.

[38] A. Rutkowska-Ziarko and Ch. Pyke, The development of downside accounting beta as a measure of risk, Econ. Bus. Rev. 3(4) (2017), 55-65.

[39] A.D. Roy, Safety first and the holding of assets, Econometrica, 20(3) (1952), 431-449.

[40] H.M. Srivastava and P.W. Karlsson, Multiple Gaussian Hypergeometric Series, Wiley, New York, 1985.

[41] H.M. Srivastava, M.I. Qureshi, K.A. Quraishi and R. Singh, Applications of Some Hypergeometric Summation Theorems Involving Double Series, J. Appl. Math. Stat. Inform. 8(2) (2012), 37-48.

[42] S.V. Stoyanov, S.T. Rachev and F.G. Fabozzi, Sensitivity of portfolio VaR and CVaR to portfolio return characteristics, Ann. Oper. Res. 205 (2013), 169-187.

[43] M. Tahir, Q. Abbas, S. Sargana, U. Ayub and S. Saeed, An investigation of beta and downside beta based CAPM-case study of Karachi stock exchange, Amer. J. Sci. Res. 85 (2013), 118-135.

[44] M. Wallmeier and M. Diethelm, Multivariate downside risk: normal versus variance gamma, J. Futures Mark. 32 (2012), $431-458$. 